\section{La gestión de la seguridad y la salud en el trabajo: acción más que retórica}

Por Yuber Liliana Rodríguez-Rojas, Ph. D. ${ }^{1}$

1 Profesional en fisioterapia. Magíster en Salud y Seguridad en el Trabajo de la Universidad Nacional de Colombia. Doctora en Administración de la Universidad de Celaya. Docente de la Maestría en Calidad y Gestión Integral. Editora de la Revista Signos. Investigación en Sistemas de Gestión. Convenio Universidad Santo Tomás-Icontec. Correo electrónico: yuberrodriguez@usantotomas.edu.co

\section{Generalidades de la gestión de la seguridad y salud en el trabajo}

Colombia decidió asumir un nuevo reto, incorporar los sistemas de gestión de la seguridad y salud en el trabajo en las organizaciones. Aunque los sistemas de gestión son una herramienta que usan las organizaciones para dar una estructura a la seguridad y salud en el trabajo (SsT) y, se ha evidenciado que, estos conllevan a efectos positivos en la gestión de las organizaciones y en la seguridad y salud de los trabajadores, no son suficientes ante las variaciones que tiene el trabajo en la actualidad, derivado de las nuevas modalidades en la prestación de servicios, el crecimiento de la complejidad organizacional y los riesgos laborales emergentes. Un ejemplo de ello, son las nuevas startups, en las cuales el negocio se soporta en las tecnologías de la información y la comunicación y, se basan en la economía colaborativa.

El estudio adelantado por Jilcha y Kitaw (2017) demuestra que la innovación en la sst contribuye al desarrollo sostenible de las organizaciones, a través de personas sanas, de lugares de trabajo seguros, de la generación de ambientes controlados que permitan reducir la accidentalidad y, por ende, los costos asociados y un mejor conocimiento de la seguridad y la salud por parte de los trabajadores. Estos investigadores concluyeron que los enfoques de mejora y de innovación en la ssT son el camino hacia el desarrollo sostenible de las organizaciones.

En este sentido, se requiere que las organizaciones superen los esquemas tradicionales de la gestión y generen procesos de innovación de la ssT. Esta expresión es entendida como un elemento clave, que permite aumentar la competitividad de las empresas y mejorar la calidad de vida de la población trabajadora. 
Lo anterior, implica la incorporación de nuevas modalidades de interacción con los trabajadores, con la mediación de las tecnologías de la información y de la comunicación; estrategias como la gamificación y la simulación, recopilación de información en tiempos más cercanos a lo real, a través de aplicativos electrónicos para la toma de decisiones oportunas, además de la innovación aplicada a los equipos, instrumentos y elementos de trabajo.

\section{Innovación de la sST y clima de innovación como estrategias de competitividad organizacional}

La innovación es considerada como una de las ventajas competitivas de las organizaciones; por ello, es un aspecto esencial para transformar las prácticas de gestión y su éxito depende de la forma en la cual se gestionan las actividades de innovación al interior de las organizaciones. De acuerdo con la OCDE, "es la introducción producto (bien o servicio) nuevo o mejorado, de un proceso, de un nuevo método organizativo o de comercialización, en las prácticas internas de la empresa, la organización del lugar del trabajo o las relaciones exteriores" (Minciencias, s. f., párr. 1). Es importante anotar que, el liderazgo, el apoyo y las expectativas gerenciales, el nivel de formación y el estilo de resolución de problemas tienen una relación directa con el comportamiento innovador de los individuos; por consiguiente, se requiere abordar, tanto las condiciones organizacionales como individuales de los trabajadores, para lograr innovar en la SST.

Aunado a ello, se ha encontrado que un clima laboral positivo contribuye en la motivación y la creatividad de los trabajadores; por lo tanto, se requiere incrementar el compromiso de los líderes, la salud laboral, la satisfacción de los trabajadores, entre otros aspectos, para fomentar un clima de innovación. De acuerdo con las reflexiones de Duque (2015), la innovación debe ser respaldada por acciones que permitan tener un ambiente en el cual las personas se sientan cómodas con sus innovaciones y generar un cambio organizacional sostenido, en otras palabras, fomentar un clima propicio para innovar. Se requiere entonces que el concepto de innovación sea visto en doble vía; es decir, tanto la innovación para transformar las prácticas de la SST y su gestión como el clima para innovación en los productos (bien o servicio) derivados de la actividad económica de la organización, que contribuyen a la sostenibilidad y competitividad de la organización.

Cabe destacar que, aunque la innovación parece la solución para incrementar la competitividad de las organizaciones y para superar las dificultades detectadas en la gestión de la seguridad y salud en el trabajo (GSST), es necesario reconocer las barreras físicas, económicas y temporales que conlleva. En esta dirección, se requiere de estrategias para innovar y para superar las barreras que se presentan dentro de las organizaciones.

Por otra parte, al revisar los planes de desarrollo nacionales de sst, de países líderes en este campo; se observa que, de acuerdo con lo establecido en la "Estrategia nacional de desarrollo de la vida laboral para 2020" de Finlandia (Ministry of Labor of Finland, 2012), el trabajo se ha transformado, tanto en los lugares donde se opera como en la estructuración de las tareas, incluso la producción está descentralizada y distribuida globalmente, adicionalmente, se enfrenta el envejecimiento de las poblaciones. Se ha encontrado que la economía se soporta en la productividad y la tasa de participación en el trabajo, es por ello, que se requiere mejorar las condiciones de trabajo y proveer oportunidades de aprendizaje y desarrollo en el trabajo para fortalecer el capital social. A la vez aportar a la cultura de trabajo (valores, 
actitudes, hábitos y costumbres), las condiciones de salud, la seguridad, la gestión y ritmo de trabajo y las buenas prácticas de gestión. Se reconoce como una de las fortalezas de Dinamarca en comparación con Finlandia y Estados Unidos la movilidad del personal y el hecho de no enfocarse en la estructura jerárquica, facilita la participación de clientes y del personal en la innovación.

$\mathrm{Al}$ igual que en Colombia, una de las problemáticas de Finlandia son los desórdenes musculoesqueléticos, de tejido conectivo y la salud mental. Aunque en este país se incorporan rápidamente nuevos modelos operativos y métodos de producción, la fuerza de trabajo tarda en adaptarse a los cambios de la vida laboral.

Aunado a ello, el cambio tecnológico, basado en las tecnologías de la información y la comunicación, ha desencadenado cambios en la producción y en el pensamiento organizacional $y$, se ha generado mayor autonomía en el personal y un mayor grado de libertad en la gestión de sus tareas, además, el trabajo se lleva a cabo de manera más independiente lo que genera mayor responsabilidad por los resultados.

En atención a este contexto, las áreas en las cuales se enfoca la estrategia de desarrollo de la vida laboral para los lugares de trabajo son: la innovación y productividad, la confianza y cooperación, la salud y bienestar en el trabajo y una fuerza laboral competente. Por lo tanto, es indiscutible que se requiere de nuevas formas de administrar, organizar y realizar el trabajo, del desarrollo de estrategias que apoyen el aprendizaje y la creatividad, junto con la capacidad de las personas para desarrollar e innovar. El éxito depende del enfoque interdisciplinario en el trabajo y la amplia capacidad tanto de las organizaciones como de los individuos para aprender, reformar, ser creativos y mantener su motivación. En otras palabras, las organizaciones requieren innovar, no solo en sus productos y servicios, también en su configuración; esto último implica articular el modelo de negocio con la gestión de la organización, crear nuevas redes de trabajo, modificar su estructura organizacional, sus procesos y sus procedimientos.

En el caso de España, las necesidades detectadas en la población trabajadora no distan de la realidad de Finlandia y de Colombia. Se requiere del abordaje de los aspectos psicosociales, de los riesgos derivados del uso de las nuevas tecnologías, de los factores emergentes derivados de aspectos sociolaborales como el envejecimiento y las nuevas tendencias de empleo. En este sentido, plantea como una de sus líneas estratégicas potenciar y revalorizar la Red de Institutos Públicos de Investigación en Seguridad y Salud en el Trabajo, fortalecer la cooperación para optimizar la $\mathrm{I}+\mathrm{D}+\mathrm{i}$ en ssT; además de promover la generación de conocimiento en SST (Ministerio de Empleo y Seguridad Social de España, 2015).

En concreto, al considerar las transformaciones actuales de los esquemas de trabajo descritos, la transformación organizacional global y la orientación que brinda la Iso 45001:2008 para la prevención de los accidentes de trabajo y las enfermedades laborales, así como su intención de alcanzar la integración del SG-SST en los procesos de negocio de las organizaciones y de fomentar una cultura de seguridad y salud en el trabajo, se ratifica la innovación como un elemento esencial de la SST.

\section{Avances desde la investigación y la innovación en la GSST en la USTA}

Al respecto, la Universidad Santo Tomás, a través de su Maestría en Calidad y Gestión Integral realizó un proyecto de investigación titulado "Métodos de evaluación para la toma de decisiones en la Gestión de la Seguridad y Salud en el Trabajo", en el cual se diseñó 
una herramienta diagnóstica de la GSST para 26 organizaciones de los sectores comercio (2), construcción (1), industria (2), servicios (11) y público (10) ubicadas en Bogotá, Colombia.

Figura 1. Estructura de la herramienta diagnóstica de la GSST

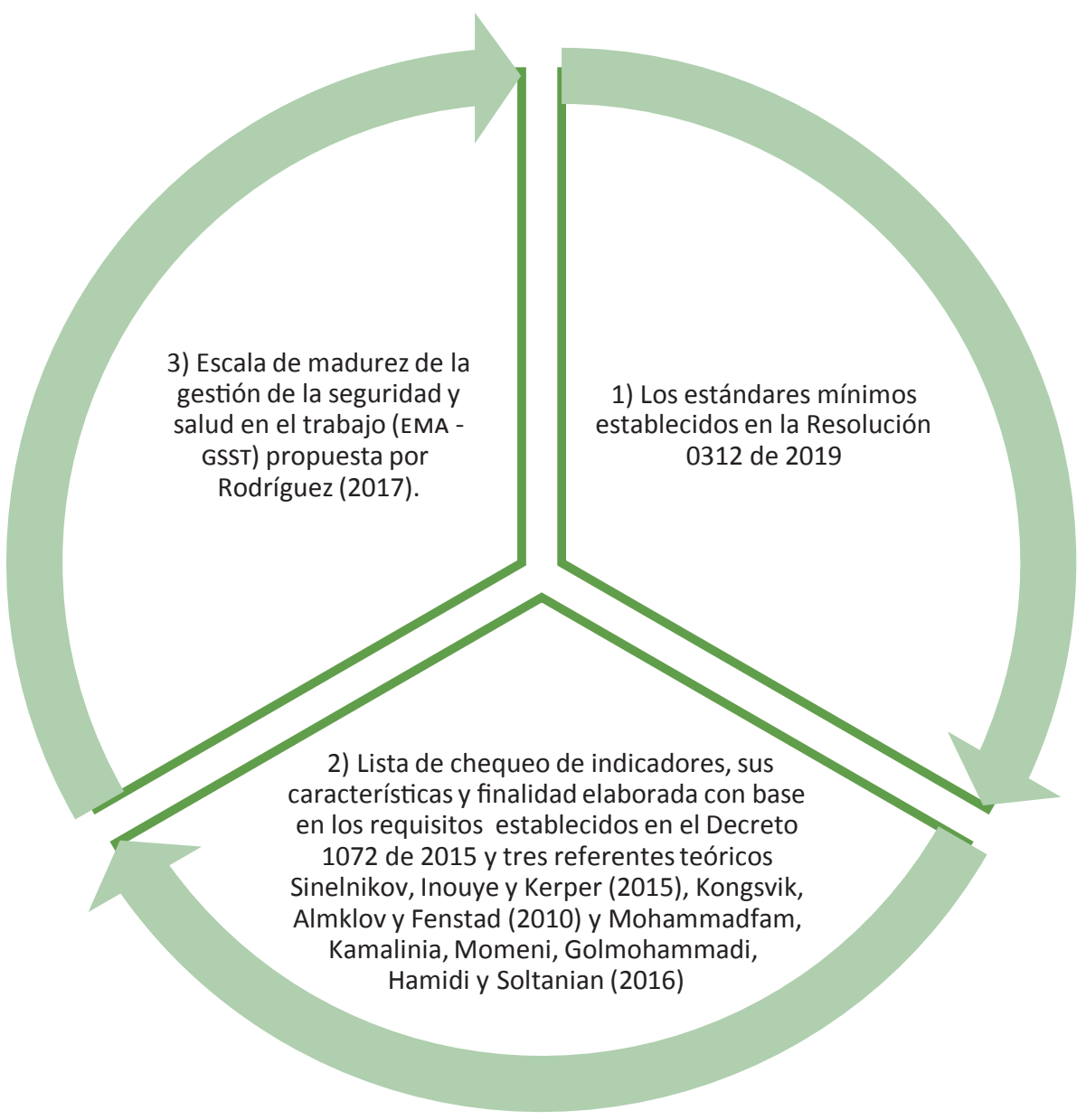

Fuente: elaboración propia.

Este proyecto permitió ratificar la aplicabilidad, validez y confiabilidad del instrumento y la escala de madurez de la gestión de la SST propuestas por RodríguezRojas (2017) en las organizaciones estudiadas. Además, brinda a estas organizaciones criterios objetivos y cuantificables para la medición de la madurez de la GSST, además apoya la identificación de oportunidades de mejora en la gestión. Asimismo, favorece la medición periódica de la GSST, dado que su aplicación es sencilla, rápida y trazable. De igual manera, provee información que ayudará a la organización a 
fijar metas en SST alcanzables a corto, mediano y largo plazo, que sean acordes con el enfoque de sst con el que se cuente.

La innovación generada con este ejercicio corresponde a la evaluación de la ggst, a partir de la triangulación de los instrumentos que constituyen una herramienta diagnóstica de la GSST construida en el proyecto. La evaluación incluye el diagnóstico inicial de la gestión de la seguridad y salud en el trabajo de la organización, de acuerdo con los requerimientos del Decreto 1072 de 2015, la Resolución 0312 de 2019 y la Escala de Madurez de la Gestión de Seguridad y Salud en el trabajo propuesta por Rodríguez-Rojas (2017).

\footnotetext{
Referencias

Duque, E. (2015). Clima de innovación para la innovación. Suma de Negocios, 6, 125-129.

Jilcha, K., \& Kitaw, D. (2017). Industrial occupational safety and health innovation for sustainable development. Engineering Science and Technology, an International Journal, 20(1), 372-380. DOI: 2048/10.1016/j.jestch.2016.10.011

Minciencias. (s. f.). Innovación empresarial [página oficial]. Bogotá. Recuperado de https://minciencias.gov.co/portafolio/innovacion/empresarial-

Ministerio de Empleo y Seguridad Social de España. (2015). Estrategia Española de Seguridad y Salud en el Trabajo 2015 -202O [en línea]. Recuperado de http://www.insht.es/InshtWeb/Contenidos/Documentacion/ESTRATEGIA\%20SST\%2015_20.pdf

Ministry of Labor of Finland. (2012). National Working Life Development Strategy to 2020 [en línea]. Recuperado de http:// www.tyoelama2020.fi/files/104/Strategy_2020.pdf

Rodríguez-Rojas, Y. (2017). Evaluación de la madurez de la gestión de la seguridad y salud en el trabajo en universidades con acreditación de alta calidad multicampus de Bogotá (Disertación doctoral). Universidad de Celaya, Celaya, México.
} 